\title{
Ocular prostheses in the last century: a retrospective analysis of 8018 patients
}

A Modugno' ${ }^{1}$, F Mantelli², S Sposato ${ }^{3}$, C Moretti ${ }^{3}$, A Lambiase $^{3}$ and S Bonini ${ }^{3}$

\begin{abstract}
Purpose To analyze the clinical characteristics of patients with ocular prosthesis to assess the causes of eye loss in the different genders and age groups, and their incidence over the years.

Methods We retrospectively examined the rates of ocular prosthesis application and related causes in the period from 1927 to 2011 in a referral center in Rome, Italy, and compared them over time. We also compared the results within the population in terms of age and gender.

Results Of 8018 ocular prosthesis wearers, $63 \%$ were males and $37 \%$ were females, with a mean age of 29 years. The most frequent cause of ocular prosthesis application was a traumatic event (54\%), with work-related eye injuries being the most frequent single cause of ocular trauma reported. Other frequent causes were end-stage ocular diseases, tumors, and malformations, without significant differences in gender. Tumors and malformations showed a slight increasing trend over time, while end-stage ocular diseases and work-related injuries remained unchanged, and other traumatic events decreased.

Conclusion The constantly high frequency of ocular prosthesis application for work-related injuries and end-stage ocular diseases suggests that preventive measures for these events have not been addressed accordingly, and might represent a neglected public-health issue. Eye (2013) 27, 865-870; doi:10.1038/eye.2013.97; published online 17 May 2013
\end{abstract}

Keywords: malformation; ocular prosthesis; trauma; tumor; work-related injury; artificial eye

\section{Introduction}

The application of an ocular prosthesis is required to replace an eye following an enucleation, evisceration, orbital exenteration, or is applied over the phthisis bulbi or a discolored blind eye of near-normal size to improve the cosmetic appearance. In fact, the loss of an eye is an immediate trigger to the physical and emotional stress caused by personal, familiar, and societal reactions to the resulting facial disfigurement. Replacing the lost eye with an ocular prosthesis aims at promoting physical and physiological healing, and improving social acceptance. ${ }^{1}$

The earliest attempt at manufacturing an ocular prosthesis dates back to as early as 4800 years ago, and several further attempts have been made in the following centuries using materials ranging from precious stones to copper, bronze, and gold in the shrunken socket. ${ }^{2,3}$ However, it was not until the sixteenth century that the first actual ocular prosthesis was designed by Ambroise Paré, a French doctor, who named it 'emblepharon'. His first prosthesis was made of gold and silver, but he later improved his first design using glass and porcelain, which was a great step forward and resulted in the use of shell-type prostheses instead of spheres. ${ }^{4}$ Glass remained the most popular material until the introduction of synthetic materials in the twentieth century. ${ }^{5}$

Currently, the main causes for the need of ocular prosthesis include tumors, congenital defects and malformations, irreparable trauma, and end-stage eye diseases. The former are the most common etiologies in children, while the latter are more common in adults. Other common etiologies include severe ocular diseases leading to blindness associated with uncontrolled pain or unpleasant cosmetic appearance, such as intraocular infections or post-surgical complications. ${ }^{1}$

In spite of their widespread distribution among populations of all ages, from children to the elderly, a register of patients with ocular
${ }^{1}$ Ocularistica Italiana of Rome, Rome, Italy

${ }^{2}$ IRCCS Fondazione Bietti of Rome, Rome, Italy

${ }^{3}$ Department of Ophthalmology, Campus Bio-Medico University of Rome, Rome, Italy

Correspondence:

S Bonini, Department of Ophthalmology, Campus Bio-Medico University of Rome, Via A. del Portillo, 200, Rome 00128, Italy. Tel: + 39 06-225419185; Fax: + 39 06-22541456;

E-mail: s.bonini@ unicampus.it

Received: 28 January 2013 Accepted in revised form: 23 March 2013 Published online: 17 May 2013 
prostheses is currently lacking, and scientific literature lacks a thorough assessment of the epidemiology of patients based on the cause that led to the need of an ocular prosthesis, age, and gender.

In this retrospective study we analyze the epidemiologic data of patients admitted to a leading Italian ocular prosthesis center in the last 85 years. Evaluating the variation in the incidence of different etiologies over time may prove beneficial for long-term prevention strategies aimed at reducing the socioeconomic costs and the personal burden of an eye loss.

\section{Materials and methods}

We analyzed the folders of 12000 consecutive ocular prosthesis wearers registered in our archive from 1927 to December 2011 following IRB approval by the University Campus Bio-Medico of Rome, Italy, and adhering to the tenets of the Declaration of Helsinki.

Inclusion criteria were represented by the presence in the reports of data on age and gender of patients, age of the first prosthesis application, and cause that led to the application of the ocular prosthesis. Exclusion criteria were the incomplete compilation of medical records, and the lack of clear and reliable clinical data.

The following characteristics of the patients were evaluated: (i) age; (ii) gender; (iii) side of ocular prosthesis application; (iv) age of application of the first prosthesis; (v) cause that led to the application of the prosthesis.

Once we grouped data from all patients, we performed all evaluations and analyses considering the following age groups as defined by the year of application of the first prosthesis: $0-5$ years (pre-scholar age), 6-14 years (primary school age), 15-18 years (secondary school age), and every 10 years thereafter.

The incidence of different etiologies over the years was also evaluated. Thereafter, a subgroup analysis was performed based on the etiologic classification of eye loss, and percentage of ocular prosthesis application for each etiology was expressed as a proportion of the total number of patients as reported in Table 1. Specifically, the cause of the defect was classified as: traumatic injuries, malformations, tumors, or end-stage eye diseases.

The traumatic injuries were further divided into accidents (road accidents, household accidents, other accidents) and work-related injuries.

Tumors were further divided into melanoma, retinoblastoma, and other types of tumor (including gliomas and other tumors).

End-stage eye diseases were further classified as anterior- or posterior-segment diseases (including corneal perforations and melting, retinal detachment, endophthalmitis of various types, end-stage glaucoma, post-surgical complications).
Table 1 Number of patients enrolled from 1927 to 2011

\begin{tabular}{rrrccr}
\hline & Total & Trauma & $\begin{array}{c}\text { End-stage } \\
\text { eye diesase }\end{array}$ & Malformations & Tumors \\
\hline $1927-1930$ & 38 & 28 & 10 & 0 & 0 \\
$1931-1940$ & 150 & 103 & 39 & 0 & 8 \\
$1941-1950$ & 511 & 435 & 62 & 6 & 8 \\
$1951-1955$ & 296 & 234 & 38 & 9 & 15 \\
$1956-1960$ & 330 & 262 & 40 & 8 & 20 \\
$1961-1965$ & 375 & 254 & 69 & 18 & 34 \\
$1966-1970$ & 434 & 300 & 60 & 22 & 52 \\
$1971-1975$ & 481 & 317 & 75 & 25 & 64 \\
$1976-1980$ & 417 & 273 & 60 & 29 & 55 \\
$1981-1985$ & 461 & 296 & 68 & 28 & 69 \\
$1986-1990$ & 437 & 248 & 75 & 43 & 71 \\
$1991-1995$ & 592 & 285 & 151 & 69 & 87 \\
$1996-2000$ & 791 & 313 & 238 & 78 & 162 \\
$2001-2005$ & 1445 & 573 & 444 & 130 & 298 \\
$2006-2011$ & 1260 & 409 & 478 & 147 & 226 \\
\hline
\end{tabular}

Data retrieved as specified above were also statistically evaluated to identify changes in the causes of eye loss and need of ocular prosthesis over time in the last century. All analyses were performed in SPSS software (SPSS 15, Chicago, IL, USA), with a $P$ value $<0.05$ considered significant.

\section{Results}

A total of 8018 patients with ocular prosthesis were included in this study. Our population consisted of 2939 females $(36.66 \%)$ and 5079 males $(63.34 \%)$, with an average of 29.32 years (SD 32.1, median of 25 years, range 0-95 years).

The distribution of our patients in different age groups was as follows: 1433 patients $(17.87 \%)$ from 0 to 5 years, 1242 patients $(15.49 \%)$ from 6 to 14 years, 589 patients (7.35\%) from 15 to 18 years, 1329 patients (16.58\%) from 19 to 30 years, 919 patients (11.46\%) from 31 to 40 years, 765 patients $(9.54 \%)$ from 41 to 50 years, 664 patients $(8.28 \%)$ from 51 to 60 years, 580 patients $(7.23 \%)$ from 61 to 70 years, 375 patients $(4.68 \%)$ from 71 to 80 years, 117 patients $(1,46 \%)$ from 81 to 90 years, 5 patients $(0.06 \%) 90$ years of age or older.

No significant differences in age of prosthesis application were observed between genders, except for the age groups of 6-14 years and 19-30 years that revealed a significantly higher number of males.

\section{Cause of prosthesis application}

The traumatic etiology was by far the most common, accounting for more than half of all cases with 4330 patients of our study population (54\%). Other causes included end-stage eye diseases in 1907 patients 
(23.78\%), tumors in 1169 patients $(14.58 \%)$, and malformations in 612 patients $(7.63 \%$ ) (Figure 1). Ocular prostheses in our study population were applied following enucleation in $45.5 \%$, following evisceration in $23.7 \%$, and over an atrophic/sub-atrophic bulb or congenital microphthalmia in $30.8 \%$.

\section{Findings of a subgroup analysis}

Trauma Traumatic injuries (eg, work-related accidents, playground accidents in kids, car crashes, fireworks, domestic accidents) represent the first cause of eye bulb loss leading to the need of an ocular prosthesis, with 3335 males $(77 \%)$ and 995 females $(23 \%)$ undergoing prosthesis application following an ocular trauma. The mean age was $28.6 \pm 17.9$. We did not observe statistically significant differences on the side affected, with 2214 patients who had the trauma in the right eye and 2087 in the left eye. Only 29 patients had a bilateral injury.

Among them, work-related accidents represent the most frequent kind of ocular trauma leading to an ocular prosthesis application, with a total of $813(10.1 \%)$ cases in our study population. Work-related accidents were more frequent in males (765 males vs 48 females, corresponding to 94 vs 6\%) and in patients aged between 19-40 years (461 patients, corresponding to $57 \%$ of the total work-related accidents), with a mean age of $34.8 \pm 14.3$.

Considering all the other kinds of trauma (ie, nonwork-related) grouped together, they appeared to happen more frequently in males (2570 patients, $73.1 \%$ ) than in females (947 patients, 26.9\%). The average age of these patients was $26.2 \pm 18.8$ years, with two distinct peaks of frequency: $25.2 \%$ in children (6-14 years of age) and $20.8 \%$ in young adults (19-30 years of age).

The overall incidence of traumatic events decreased gradually, with the highest peak in the 1950s and the lowest in 2010. A subgroup analysis showed a similar trend of ocular prosthesis application over the years in patients who had non-work-related accidents, while no decrease in the incidence of work-related accidents was observed, with no statistically significant differences among all the decades examined (Figure 2).

End-stage eye diseases The total number of ocular prosthesis wearers who had an end-stage eye disease was 1907 (23.78\%), including patients with anterior(corneal ulcers, perforations, melting) and posteriorsegment disease (retinal detachment, glaucoma, endophthalmitis), and surgical complications. In these 1907 patients no significant differences in gender were noted, with 901 males (47.2\%) and 1006 females (52.8\%) in our study population. The mean age of the patients was $39.5 \pm 24.6$ years, and patients in the $61-70$ years age group represented the majority of ocular prostheses wearers for end-stage eye disease (273 patients, $14.3 \%$ ). However, we did not find statistically significant differences with other age groups. We also did not observe statistically significant differences on the side affected, with 942 patients who had an end-stage eye

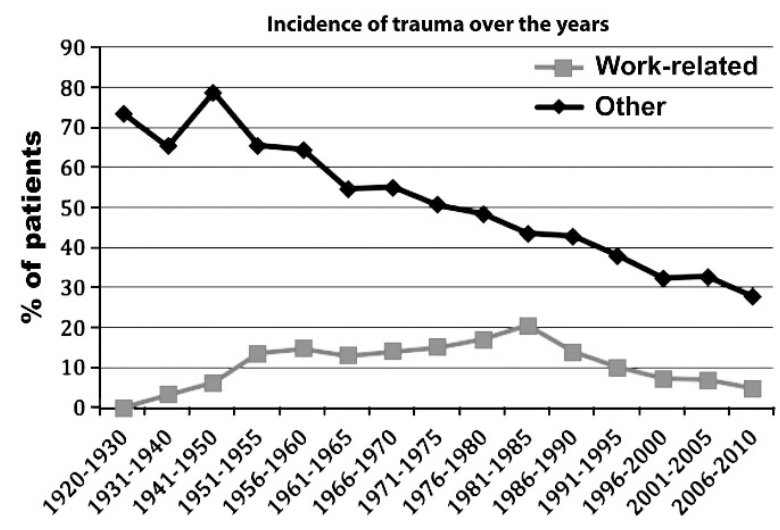

Figure 2 Incidence of traumatic ocular injuries over the analyzed decades.

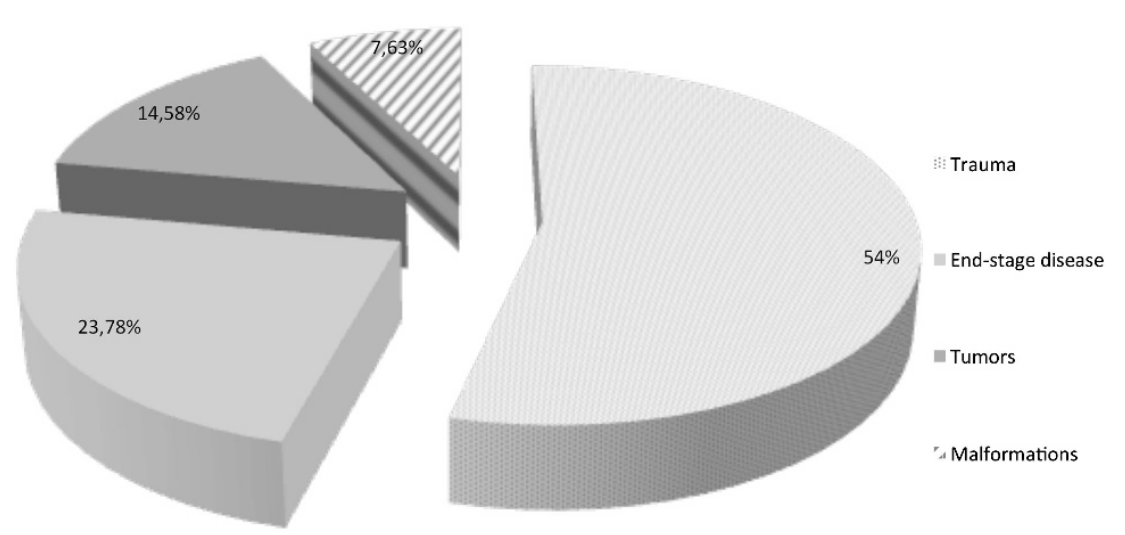

Figure 1 Causes of ocular prosthesis application in the study population. 
disease in the right eye and 905 in the left eye. Only 60 patients had a bilateral disease.

A subgroup analysis of the end-stage eye disease population failed to demonstrate any significant difference in the primary cause that led to the need of an ocular prosthesis application, classified as anterior- or posterior-segment related. Specifically, in our study population $52 \%$ of all patients received an ocular prosthesis following an anterior ocular disease or surgical complication from an anterior ocular disease, and $48 \%$ of all patients received an ocular prosthesis following a posterior ocular disease or surgical complication from a posterior ocular disease.

The incidence of end-stage eye diseases did not change significantly among all the decades examined.

Tumors Among the 1169 patients in our records who needed an ocular prosthesis following an ocular tumor, $603(51.6 \%)$ had a retinoblastoma, $520(44.5 \%)$ had a melanoma, and $46(3.9 \%)$ had other types of tumor (optic nerve glioma, meningioma, ocular adnexas' tumors). The mean age for retinoblastoma patients was $2.5 \pm 5.2$ years, for melanoma patients $56.2 \pm 38.4$ years, and for patients with other types of tumor $38.8 \pm 25.1$ years. While retinoblastoma's incidence was significantly higher in the $0-5$ years age group $(P<0.001)$, melanoma's incidence was more widespread with the majority of patients affected (65\%) aged between 40 and 80 years.

No significant differences were observed in gender distribution, with 545 males (47\%) and 624 females (53\%), unrelated to the type of tumor. We also did not observe statistically significant differences on the side affected, with 584 patients who had the tumor in the right eye and 546 in the left eye. Only 39 patients had a bilateral tumor (all retinoblastomas).

The analysis of the incidence of prosthesis application in patients affected by an ocular tumor increased gradually over the years (Figure 3a), mainly due to an increase in the two most common ocular tumors leading to the application of an ocular prosthesis, melanoma and retinoblastoma (Figure $3 b$ ).

Malformations Among the 612 patients who required the application of an ocular prosthesis due to a congenital ocular malformation, we did not observe a statistically significant difference between males (299 patients, $48.86 \%$ of the total) and females (313 patients, $51.14 \%$ of the total). The mean age was $8 \pm 11.8$ years and the majority of patients fell into the $0-5$ years age group (389 patients, 63.6\%).

We did not observe statistically significant differences on the side affected, with 286 patients whose ocular malformation was on the right side, and 276 on the left

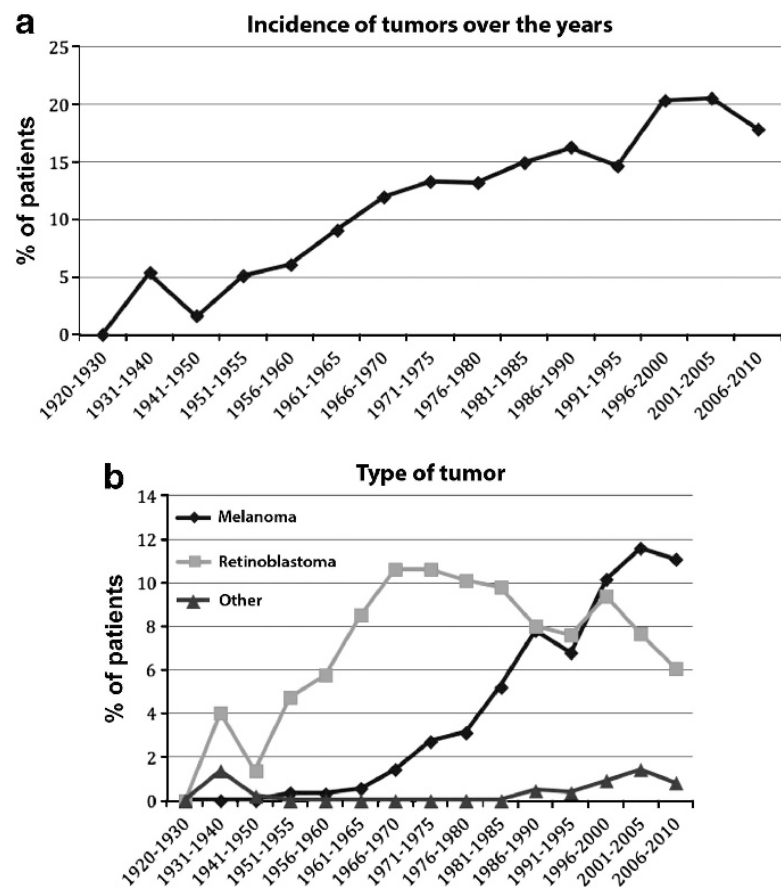

Figure 3 The incidence of prosthesis application in patients affected by ocular tumors (a) increased gradually over the years. The incidence of the different types of tumor is shown in panel (b).

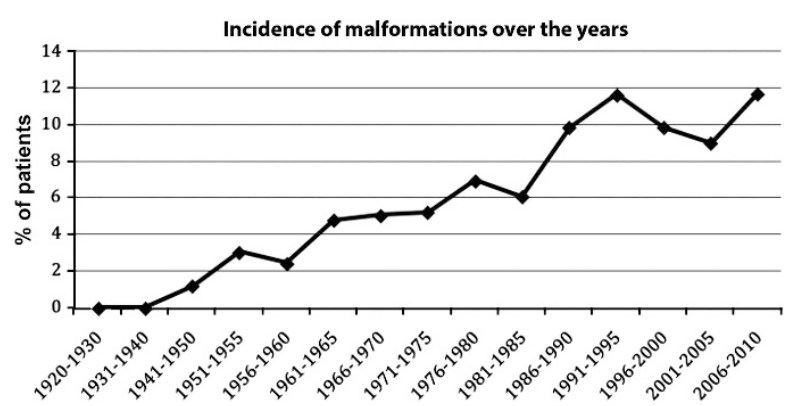

Figure 4 The incidence of prosthesis application for ocular malformations showed a gradual increase over the years.

side. Only 50 patients had a bilateral ocular malformation.

As shown in Figure 4, the analysis of the incidence of prosthesis application for ocular malformations such as anophthalmia and microphthalmia revealed a gradual increase over the decades.

\section{Discussion}

In this study we describe the largest population of patients with ocular prosthesis analyzed so far in scientific literature, reporting data on age, gender, and cause of ocular prostheses application.

Males in work age (19-60 years) represent the category at higher risk of an event leading to ocular prosthesis 
application, which may reflect the increased number of work-related injuries men face due to their typical occupation. In fact, $94 \%$ of work-related eye injuries occur in male workers, and represent more than $10 \%$ of all causes of eye loss in our population. Surprisingly, in spite of the huge policy-maker efforts in increasing safety on the workplace with several campaigns of prevention-especially since the 80 s-work-related eye injuries did not decrease over time.

Prevention of work-related injuries represents a priority for national health systems of industrialized countries due to the high costs of disability benefits, and for the substantial economic impact due to lost income and long-term medical treatments. The relevance of the social burden has also been recently highlighted by the increasing rate of lost work-time due to work-related eye injuries in US farmworkers. ${ }^{6}$ Moreover, while workrelated eye injuries are more frequent in young workers and afflict the society due to loss of occupational workforce, injuries in older workers often lead to higher levels of disability, with consequently higher costs for the health system. ${ }^{7-11}$

It is interesting to underline that while the rate of work-related trauma remained unchanged, the overall incidence of traumatic injuries decreased progressively over the years, probably due to better standards of safety and/or better prevention strategy campaigns for domestic accidents, road accident, or other kind of accidents including fire-works and sport accidents. ${ }^{12}$ Nevertheless, traumatic injuries still represent the first cause of ocular prosthesis need, with $54 \%$ of all patients in our study population and a significantly higher prevalence in males, which may be explained by a higher number of accidents of various kinds, more active games, and more dangerous sports in males. ${ }^{13-15}$

The second cause (23.78\%) for ocular prosthesis application in our study population was an end-stage eye disease or related surgical complications. ${ }^{16}$ As expected, we did not observe any significant difference in gender and side of the ocular disease, which were more frequent in the over- 60 population, probably reflecting the higher number of surgical procedures in the elderly. We did not find significant differences between anterior- and posterior-segment diseases; however, we could not further discriminate between the specific diseases, as in older clinical charts the specific disease was not recorded. Nevertheless, it remains interesting that the overall rate was unchanged in the past decades in spite of the huge progress in ocular medical and surgical approaches.

Ocular tumors represented the third most frequent cause $(14.58 \%)$ of ocular prosthesis application. Among them, retinoblastoma was the most frequent (51.6\%), which may be explained by the higher number of patients in the $0-5$ years age group, reflecting the need of a prompt application of an ocular prosthesis in children in order to develop a normal facial symmetry. ${ }^{17,18}$

Melanoma was the second most frequent tumor (44.5\%), and was also observed in adult prosthesis wearers. The distribution according to gender did not show a statistically significant difference, as already reported in scientific literature. ${ }^{19}$

The analysis of the incidence revealed a gradual increase of prosthesis application for ocular tumors, which probably reflects the progress in early diagnosis and treatment, that reduced the mortality rates of these unfortunate events. ${ }^{20,21}$

Lastly, ocular malformations represented $7.63 \%$ of our cases, without any statistically significant difference according to gender and side, and the majority of patients aged $0-5$ years (63.6\%). Interestingly, we observed an increasing number of ocular prosthesis applied in patients with ocular malformations over the years, which may reflect indirect evidence that malformative diseases followed an upward trend, or may reflect an improvement in the therapeutic scheme and patients' follow-up. ${ }^{22-24}$ In fact, while there is higher education of caregivers about the early rehabilitation of the orbital cavities with anophthalmia or microphthalmia, it cannot be excluded that we observed an actually higher rate of ocular malformations, which could be the result of increasing maternal age and increasing risk factors in the gestation period. ${ }^{25}$

To conclude, our study emphasizes the need and importance of creating a register of ocular prosthesis wearers to provide healthcare and policy makers with crucial information to actuate the necessary interventions for reducing risks, and the socio-economic and psychological impact of events leading to the application of an ocular prosthesis.

\section{Summary}

\section{What was known before:}

- In spite of the widespread distribution of ocular prostheses among populations of all ages, from children to the elderly, a register of patients with ocular prostheses is currently lacking and scientific literature lacks a thorough assessment on the epidemiology of patients based on the cause that led to the need of an ocular prosthesis, age, and gender.

\section{What this study adds:}

- In our paper we analyze the epidemiologic data of more than 8000 patients with an ocular prosthesis, evaluating the variation in the incidence of different etiologies in the last century. The data from our work, representing the largest ocular prosthesis wearers population analyzed so far in scientific literature, may prove beneficial for long-term prevention strategies aimed at reducing the socio-economic costs and the personal burden of an eye loss. 


\section{Conflict of interest}

The authors declare no conflict of interest.

\section{Disclaimer}

This paper has not been published nor submitted in full or in part elsewhere.

\section{References}

1 Artopoulou I, Mountgomery P, Wesly P, Lemon JC. Digital imaging in the fabrication of ocular prosthesis. J Prosthet Dent 2006; 95: 327-330.

2 5,000-Year-Old Artificial Eye Found on Iran-Afghan Border, The Times, 20 February 2007.

3 Roberts AC. Facial prostheses. 1st ed. Henry Kimpton: London, USA, 1971, p 4.

4 Gibson T. The prostheses of Ambroise Pare. Br J Plast Surg 1955; 8: 3-8.

5 Dyer NA. The artificial eye. Aust J Ophthalmol 1980; 8: 325-327.

6 Quandt SA, Schulz MR, Talton JW, Verma A, Arcury TA. Occupational eye injuries experienced by migrant farmworkers. J Agromedicine 2012; 17: 63-69.

7 Lewis MQ, Sprince NL, Burmeister LF, Whitten PS, Torner JC, Zwerling C. Work-related injuries among Iowa farm operators: an analysis of the Iowa Farm Family Health and Hazard Surveillance Project. Am J Ind Med 1998; 33: 510-517.

8 Jackson LL. Non-fatal occupational injuries and illnesses treated in hospital emergency departments in the United States. Inj Prev 2001; 7(Suppl 1): i21-i26.

9 Salminen S, Heiskanen M. Correlations between traffic, occupational, sports, and home accidents. Accid Anal Prev 1997; 29: 33-36.

10 Sears JM, Blanar L, Bowman SM, Adams D, Silverstein BA. Predicting work-related disability and medical cost outcomes: estimating injury severity scores from workers' compensation data. J Occup Rehabil 2012; 23(1): 19-31.

11 Foley M, Fan ZJ, Rauser E, Silverstein B. The impact of regulatory enforcement and consultation visits on workers' compensation claims incidence rates and costs, 1999-2008. Am J Ind Med 2012; 55: 976-990.
12 Cole MD, Smerdon D. Perforating eye injuries caused by darts. Br J Ophth 1988; 72: 511-514.

13 McGwin G, Owsley C. Incidence of emergency departmenttreated eye injury in the United States. Arch Opthalmol 2005; 123: 662-666.

14 Cillino S, Casuccio A, Di Pace F, Pillitteri F, Cillino G. A fiveyear retrospective study of the epidemiological characteristics and visual outcomes of patients hospitalized for ocular trauma in a Mediterranean area. BMC Ophthalmol 2008; 8: 6

15 Mattos BS, Carvalho JC. Prevalence of eye loss. 3. Study of surgical variations and their correlation with etiology and sex. Rev Odontol Univ Sao Paulo 1989; 3: 284-288.

16 Mattos BS, Carvalho JC. Prevalence of eye loss. 2. Study of age variables and correlation with etiology, sex and ophthalmic surgery. Rev Odontol Univ Sao Paulo 1989; 3: 246-252.

17 Abramson DH, Servodidio CA. Retinoblastoma in the first year of life. Ophthalmic Genet 1992; 13: 191-203.

18 Yang CP, Hung IJ, Jaing TH, Chang WH. Cancers in infancy: percent distribution and incidence rates. Acta Paediatr Taiwan 2006; 47: 273-277.

19 Singh AD, Bergman L, Seregard S. Uveal melanoma: epidemiologic aspects. Ophthalmol Clin N Am 2005; 18: 75-84.

20 Dimaras H, Kimani K, Dimba EA, Gronsdahl P, White A, Chan HS et al. Retinoblastoma. Lancet 2012; 379: 1436-1446.

21 Damato B. Does ocular treatment of uveal melanoma influence survival? Br J Cancer 2010; 103: 285-290.

22 Fryns JP, Witters I. Anophthalmia/microphtalmia associated with right lateral facial cleft/microtia. Genet Couns 2001; 12: 177-178.

23 Forrester MB. Descriptive epidemiology of anophthalmia and microphthalmia, Hawaii, 1986-2001. Birth Defects Res A Clin Mol Teratol 2006; 76: 187-192.

24 Mattos BS, Montagna MC, Fernandes Cda S, Sabóia AC. The pediatric patient at a maxillofacial service - eye prosthesis. Braz Oral Res 2006; 20: 247-251.

25 Salmeen K, Zlatnik M. The oldest gravidas: a review of pregnancy risks in women over 45. Obstet Gynecol Surv 2001; 66: 580-590. 